\title{
Methylated PTGER4 is better than CA125, CEA, Cyfra211 and NSE as a therapeutic response assessment marker in stage IV lung cancer
}

\author{
YUNXIA ZHANG $^{1 *}$, JIANFENG HUANG $^{1 *}$, QINZHOU ZOU $^{1}$, JUN CHE $^{1}$, KAIHUA YANG $^{1}$, \\ QIANG FAN $^{1}$, DANQI QIAN ${ }^{1}$, JIA WU ${ }^{1}$, ERWEN BAO $^{1}$, LELE SONG $^{2,3}$ and FUZHENG ZHANG ${ }^{1}$ \\ ${ }^{1}$ Department of Radiotherapy, The Affiliated Hospital of Jiangnan University, Binhu, Wuxi, Jiangsu 214062; \\ ${ }^{2}$ Department of Radiotherapy, The Eighth Medical Center of The Chinese People's Liberation Army General Hospital, \\ Haidian, Beijing 100091, ${ }^{3}$ HaploX Biotechnology, Co., Ltd., Shenzhen, Guangdong 518057, P.R. China
}

Received July 21, 2019; Accepted January 30, 2020

DOI: 10.3892/ol.2020.11434

\begin{abstract}
Real-time assessment of therapeutic response in patients with advanced lung cancer presents a major challenge throughout the treatment process. Currently, computed tomography imaging is often used; however, it is radiation-based and hysteretic and is not suitable for repeated use as a real-time assessment. Blood biomarkers represent a novel solution for assessing therapeutic response in patients with advanced lung cancer. In the present study, the efficacy of a methylation marker [methylated prostaglandin E receptor 4 (mPTGER4)] and four protein markers [carcinoma antigen 125 (CA125), carcinoembryonic antigen (CEA), cytokeratin 19-fragments (cyfra21-1) and neuron-specific enolase (NSE)] were simultaneously evaluated to determine their potential in facilitating therapeutic response monitoring as well as their prognostic values in patients with stage IV lung cancer. The results indicated that, following treatment, the blood levels of methylated PTGER4 and NSE had significantly decreased, and mPRGER4, CA125, CEA and NSE exhibited a significant decrease in percentage level. Since mPTGER4 exhibited a higher rate of positive detection prior to therapy, and a greater response of sensitivity to therapy compared to the protein markers, it may
\end{abstract}

Correspondence to: Professor Fuzheng Zhang, Department of Radiotherapy, The Affiliated Hospital of Jiangnan University, 200 Huihe Road, Binhu, Wuxi, Jiangsu 214062, P.R. China

E-mail:wxsyzfz@163.com

Dr Lele Song, Department of Radiotherapy, The Eighth Medical Center of The Chinese People's Liberation Army General Hospital, 17 Heishanhu Road, Haidian, Beijing 100091, P.R. China

E-mail: songlele@sina.com

*Contributed equally

Key words: lung cancer, prognosis, survival, prostaglandin $\mathrm{E}$ receptor 4, carcinoma antigen 125 , carcinoembryonic antigen, cytokeratin 19-fragments, neuron-specific enolase represent an improved marker for the monitoring of therapeutic response. The efficacy of the markers in predicting the overall survival (OS) rate of patients with stage IV lung cancer was also assessed. Results from the follow-up of patients (up to 891 days) revealed that the blood levels of mPTGER4, CA125 and NSE before treatment were able to predict overall survival (OS) rate. Additionally, the percentage change in expression levels of CA125, CEA and NSE was also able to predict the OS rate. In conclusion, the present results indicate that mPTGER4 represents an improved biomarker for monitoring therapeutic efficacy compared with CA125, CEA, Cyfra21-1 and NSE. In predicting the long-term survival of patients with stage IV lung cancer; however, the pre-treatment levels of mPTGER4, CA125 and NSE and the percentage changes of CA125, CEA and NSE may be used as the markers.

\section{Introduction}

Lung cancer is the most commonly diagnosed cancer and the leading cause of cancer-associated mortality in China and globally (1). Low-dose computed tomography has been recommended as the primary method for screening lung cancer (1). Multiple in vitro diagnostic methods for lung cancer screening and early detection, including next-generation sequencing and blood-based circulating tumor DNA (ctDNA) assays, are currently under development with promising application prospects (2-7). However, there are few effective blood-based tests for assessing the therapeutic response or predicting the prognosis of patients with lung cancer (8-10). The sensitivity of clinically used protein markers, such as carcinoma antigen 125 (CA125), carcinoembryonic antigen (CEA), cytokeratin 19-fragments (cyfra21-1) and neuron-specific enolase (NSE) is not high enough for effective screening (2-10). In addition, patients with negative test results prior to treatment are unable to be assessed using protein markers, which are generally more sensitive to advanced lung cancer than early-stage lung cancer (8-10). Therefore, protein markers are more suitable to be used as recurrence indicators, rather than response monitoring markers. However, CT imaging, despite being a widely-used, non-invasive method for therapeutic response assessment, is 
unable to be used routinely due to radiation, hence renders the immediate detection of tumors changes impossible. Currently, effective methods for response monitoring and prognosis prediction in lung cancer therapy are lacking.

Methylated prostaglandin E2 receptor EP4 subtype (mPTGER4) was recently reported as a methylation marker for the early detection of lung cancer. To the best of our knowledge, the only two studies investigating the application of mPTGER4 as a marker involve the use of this marker in the differential diagnosis of benign and malignant lung diseases $(11,12)$. Other diagnostic and therapeutic applications of mPTGER4 have not yet been reported. In the aforementioned two reports, mPTGER4 was used in conjunction with methylated short stature homeobox 2 (mSHOX2) to differentiate between benign and malignant lung diseases, with an enhanced sensitivity and specificity of 67 and 90\%, respectively compared with mPTGER4 or mSHOX2 alone $(11,12)$. Methylation markers generally exhibit stage-dependent detection sensitivity, which is typically higher in advanced cases. In early-stage patients, however, the detection sensitivity is determined by the actual performance of markers in early detection and differential diagnosis (11-16). For patients with advanced disease, methylation markers can potentially be used for therapeutic response monitoring, due to their high positive detection rate (PDR) in advanced stage cancers, as well as their sensitivity to changes in tumor burden following treatment $(15,16)$. Conversely, protein markers are not as potent as methylation markers, due to their unsatisfactory PDR even in advanced cancer, thus limiting their use in monitoring techniques $(8-10,17)$. However, the specificity of protein markers is generally satisfactory $(18,19)$, therefore, they are often better indicators of recurrence instead.

In the present study, the efficacy of methylated PTGER4 was systematically assessed and compared with four clinically-used protein markers (CA125, CEA, Cyfra211 and NSE) in therapeutic response monitoring and survival prediction in patients with stage IV lung cancer. Blood samples were analyzed before and after two cycles of treatment, and the effectiveness of these markers in monitoring the therapeutic response was assessed. Concurrently, follow-up was performed on the patients for up to 891 days, and the efficacy of the markers in predicting survival based on pre- and post-therapeutic blood levels was compared, as well as the relevant percentage changes. It was revealed that methylated PTGER4 was better than protein markers in therapeutic response monitoring, while methylated PTGER4, CA125, CEA and NSE were useful for predicting the overall survival rate. The present study validated methylated PRGER4 as a potential marker for future continuous response monitoring and prognosis prediction in patients with stage IV lung cancer.

\section{Materials and methods}

Ethics. The protocol of the present clinical study was approved by the ethics committees of the affiliated hospital of Jiangnan university and the eighth medical center of the Chinese People's Liberation Army general hospital prior to sample collection. Written informed consent was obtained from all subjects, and information on the intended usage of plasma and test results was provided to all subjects.
Study design, patients and therapy. The current study was designed and implemented in the Affiliated Hospital of Jiangnan University (Jiangsu, China) and The 8th Medical Center of the Chinese PLA General Hospital (Beijing, China). A methylated PTGER4 assay was used as previously reported by Weiss et al (11). Clinical status was determined prior to blood collection for the methylated PTGER4 assay, and blood samples were obtained from all subjects who met the selection criteria. The main inclusion criteria: adults $>18$ years old with complete clinicopathological information and confirmed diagnosis of lung cancer by imaging examination (including MRI, CT, etc.) and/or subsequent pathological examination. The main exclusion criteria include: pregnancy, history of any cancer, or history of therapy for any cancer. Subjects with incomplete information were also excluded. All technicians were blinded to the clinical information of subjects, and patients' test results and corresponding clinical information were only revealed after all tests were finished. All patients were $\geq 18$ years old with no previous history of cancer and both male and female patients were included. All lung cancer subtypes, including small-cell lung cancer (SCLC) and non-small cell lung cancer (NSCLC) were included. Adenocarcinoma (ADC) and SCLC were the most prevalent subtypes in the present study (Table I). A total of 146 subjects were recruited in the current study, and all patients had primary lung cancer without any history of treatment. The population comprised 30 patients at stage I, 29 at stage II, 36 at stage III and 51 at stage IV (Table I). Tumors were staged in accordance with the NCCN guidelines (13). Determination of stage was achieved by at least two independent pathologists via pathological examination of biopsies from needle aspiration. All patients at stage IV were diagnosed using imaging and pathological examinations, in which $\geq 1$ primary lung cancer lesion and $\geq 1$ confirmed distal metastasis were discovered. First-line standard chemotherapy, combined radio- and chemotherapy or tyrosine kinase inhibitor (TKI)-based targeted therapy was used to treat stage IV patients. The diameter of the primary tumors was assessed based on RECIST 1.1 criteria, and correlated with the level of mPTGER4 (20).

Population size estimation. The equation for known positive detection rate was used for Population size estimation: $\mathrm{n}=\mathrm{Z}^{2} \mathrm{x}[\mathrm{p}(1-\mathrm{p})] / \mathrm{E}^{2} . \mathrm{Z}$ is a statistical parameter $(\mathrm{Z}=1.96$ for $95 \% \mathrm{CI})$ and $E$ represented the error (10\% was selected in the present study), and $\mathrm{p}$ represented the putative positive detection rate. The E value is generally $0.05-0.1$, depending on the error that is allowed in a study. Since the effectiveness of the mPTGER4 test has been validated in previous studies $(11,12)$ and a previous pilot study, 0.1(10\%) was used in the present study. The $\mathrm{p}$ value represented the known sensitivity for the assay on lung cancer (11), and was obtained from a previous pilot study (data not shown). If the known sensitivity for stage IV lung cancer equals to 0.85 , an estimated 49 lung cancer cases were required. A total of 51 patients (32 male and 19 female; age range, 43-82 year; median age, 63) with stage IV lung cancer were included in the current study (Table I); and this total comprised 12 patients with SCLC and 39 with NSCLC. Of the patients with NSCLC, 14 exhibited epidermal growth factor receptor (EGFR)-sensitive mutations (EGFR M+) and underwent TKI-based first-line therapy and 25 patients did not have $E G F R$-sensitive mutations (EGFR M-) 
Table I. Number of enrolled subjects and demographic characteristics by diagnosis group.

\begin{tabular}{|c|c|c|c|c|c|c|c|}
\hline \multirow[b]{2}{*}{ Diagnosis group } & \multicolumn{3}{|c|}{ Sex, $n$} & \multicolumn{4}{|c|}{ Age, years } \\
\hline & Total & Male & Female & $<50$ & $50-59$ & $60-69$ & $\geq 70$ \\
\hline Overall & 146 & 95 & 51 & 20 & 51 & 48 & 27 \\
\hline \multicolumn{8}{|l|}{ Stage } \\
\hline $\mathrm{I}$ & 30 & 21 & 9 & 2 & 11 & 12 & 5 \\
\hline II & 29 & 17 & 12 & 4 & 9 & 10 & 6 \\
\hline III & 36 & 25 & 11 & 9 & 14 & 8 & 5 \\
\hline IV & 51 & 32 & 19 & 5 & 17 & 18 & 11 \\
\hline \multicolumn{8}{|c|}{ Pathological type (stage IV) } \\
\hline SCLC & 12 & 8 & 4 & 1 & 4 & 4 & 3 \\
\hline $\mathrm{ADC}$ & 35 & 21 & 14 & 4 & 11 & 12 & 8 \\
\hline $\mathrm{SC}$ & 3 & 2 & 1 & 0 & 1 & 2 & 0 \\
\hline $\mathrm{LC}$ & 1 & 1 & 0 & 0 & 1 & 0 & 0 \\
\hline
\end{tabular}

SCLC, small-cell lung cancer; ADC, adenocarcinoma; SC, squamous cell carcinoma; LC, large-cell carcinoma.

and underwent either standard chemotherapy or combined chemo- and radiotherapy.

Sample collection and storage. A $10 \mathrm{ml}$ peripheral blood sample was collected from lung cancer patients of the two participating hospitals using $10 \mathrm{ml} \mathrm{K}_{2}$ EDTA anticoagulant tubes (BD Biosciences). Sample storage and transportation followed the methods previously reported by Weiss et al (11). The first blood sample was collected prior to initiation of any therapy, and the second blood sample was collected following two cycles of therapy. One cycle of chemotherapy lasts 21 days, while the TKI therapy requires patients to take medicine daily. Therefore, the blood collection point was on the 42nd day following treatment initiation for all patients, prior to the beginning of the third cycle of therapy. The sample information was recorded in sample collection forms. Plasma samples from all participating hospitals were prepared in the individual hospitals and stored at $-20^{\circ} \mathrm{C}$ prior to delivery to the laboratory, and all assays were performed in the same laboratory $\leq 3$ weeks from the sample collection date. The sample quality was examined when the samples arrived at the medical laboratory. Samples with plasma volume $<3.5 \mathrm{ml}$, or with apparent hemolysis, high bilirubin, chylemia or visible particles/pellets were not tested and repeated blood draw was requested.

DNA extraction, qualitative PCR analysis of PTGER4 and measurement of protein marker levels. A commercial kit, Epi proLung (cat. no. M6-02-002, Epigenomics AG) was used in this study. The experiments were carried out according to the manufacturer's instructions (21) (https://www.epigenomics.com/wp-content/uploads/2018/08/IFU_0018_GB_ rev4_Instructions_for_Use_Epi_proLung.pdf). In brief, DNA extraction and bisulfite conversion were performed manually from plasma circulating DNA following the methods reported by Weiss et al (11). The bisulfite-converted DNA was assayed using an ABI7500 Fast Dx Real Time
PCR device (Thermo Fisher Scientific, Inc.). PCR was performed in triplicate with $15 \mu 1$ template DNA per well, and run for 45 cycles. The validity of each sample batch was determined on the basis of methylated PTGER4 and $\beta$-actin $(A C T B)$ threshold count $(\mathrm{Ct})$ values for the positive and negative controls. $A C T B$ was used as an internal reference to assess the integrity of each sample. The sequences of the primers and probes for PTGER4 are listed in the corresponding patents (patent numbers, EP2143807A1, EP3234184B1, AU2008207110B2 and US20090203011A1). The sequence of primers for $\beta$-actin detection used in PCR amplification were as follows: forward primer, 5'-GTGATGGAGGAGGTTTAGTAAGTT-3' and reverse primer, 5'-CCAATAAAACCTACTCCTCCСТTAA-3' and probe, 5'-ACCACCACCCAACACACAATA ACA AAC ACA-3'. The blood levels of protein markers, including CA125, CEA, Cyfra21-1 and NSE, were measured by trained technicians in the corresponding hospitals, according to the in-house procedures.

Data analysis and interpretation. Test data of the mPRGER4 assay were analyzed by calculating the $\Delta \Delta \mathrm{Ct}$ values using the $\mathrm{Ct}$ values from samples, $A C T B$ internal controls and the positive controls (14). Statistical analysis was performed and figures were plotted using GraphPad Prism software (version 5.0; GraphPad Software, Inc.). For each sample, a relative methylation value was determined using the $\Delta \Delta \mathrm{Ct}$ method adapted for DNA methylation analyses as previously described (14). In brief, $\Delta \Delta \mathrm{Ct}$ values were calculated as below: $\Delta \Delta \mathrm{Ct}_{\text {Sample }}=\Delta \mathrm{Ct}_{\mathrm{Sample}^{-}}$ $\Delta \mathrm{Ct}_{\text {Calibrator }}$, where $\Delta \mathrm{Ct}_{\text {Sample }}=\mathrm{Ct}_{\mathrm{ACTB} \text { of sample }}-\mathrm{Ct}_{\mathrm{PTGER} 4 \text { of sample }}$ and $\Delta \mathrm{Ct}_{\text {Calibrator }}=\mathrm{Ct}_{\mathrm{ACTB} \text { of calibrator }}-\mathrm{Ct}_{\mathrm{PTGER} 4 \text { of calibrator }}$.

The unpaired Student's t-test was used to compare two groups. The $\chi^{2}$ test and Fisher tests were performed when rate or percentage was compared for significance. ${ }^{*} \mathrm{P}<0.05$ represented significant changes, ${ }^{* *} \mathrm{P}<0.01$ represented highly significant changes and ${ }^{* * *} \mathrm{P}<0.001$ represented very highly significant changes. Kaplan-Meier curves were plotted 
and analyzed using Graphpad Prism software (version 5.0; GraphPad Software, Inc.) and the log-rank (Mantel-Cox) test was performed to compare the survival curves. Patients were divided into two groups in the survival analysis by the median levels of mPTGER4, CA125, CEA, Cyfra211 and NSE in the blood. As the total number of stage IV lung cancer patients was 51 in the present study, each group had 25 or 26 subjects depending on the exact median value. In the survival analysis of NSCLC patients the total number of patients was 39 and each group had 19 or 20 subjects depending on the exact median value. $\mathrm{P}<0.05$ was considered to indicate a statistically significant difference between survival curves.

\section{Results}

Methylated PTGER4 exhibits better performance as a marker in therapeutic response assessment compared with CA125, CEA, Cyfra211 and NSE. In order to investigate the performance of methylated PTGER4, CA125, CEA, Cyfra21-1 and NSE as indicators of therapeutic efficacy in patients with stage IV lung cancer, the positive detection rate (PDR) of each molecule was individually calculated. It was revealed that the PDR of methylated PTGER4 increased as the cancer stage progressed, with a high PDR reaching $78.0 \%$ in stage IV lung cancer (Fig. 1A). Meanwhile, the PDRs of CA125, CEA, Cyfra21-1 and NSE were revealed to be $46.2,48.0,52.0$ and $58.3 \%$, respectively, which were significantly lower compared with methylated PTGER4 (Fig. 1B). A higher PDR value signifies that a higher ratio of patients may benefit from the assessment, and methylated PTGER4 exhibited a significantly higher coverage of patients with stage IV lung cancer than the protein markers measured.

Blood level changes of the five markers were also measured pre- and post-treatment. Patients were divided into a PR group and SD group based on RECIST1.1 criteria (20), as detailed in Fig. 2. In the PR groups, while the levels of methylated PTGER4 and NSE significantly decreased following treatment, there were no significant difference observed between CA125, CEA and Cyfra21-1 levels. In the SD group, there was no significant difference in blood levels before and after treatment for all markers. It is worth noting that the plasma level of methylated PTGER4 decreased significantly. The average $\Delta \Delta \mathrm{Ct}$ value (mean $\pm \mathrm{SD}$ ) decreased 85.10 -fold from $-2.634 \pm 1.486$ before treatment to $-9.045 \pm 1.139$ after treatment $\left(2^{-2.634-(-9.045)}=2^{6.411}=85.1\right)$, which was a fold-change much larger than any protein marker tested. The present results indicate that methylated PTGER4 and NSE accurately reflected the therapeutic response of patients with stage IV cancer in the PR group, while other markers were not effective in reflecting such a response.

Since the level changes of the markers shown in Fig. 2 only indicates the overall change in this specific population, the normalized percentage changes in the PR and SD group were also calculated. Fig. 3 depicts a comparison of the percentage change of each marker in the PR and SD groups, normalized against the pre-therapeutic marker level. Significant differences in percentage between the PR and SD group in mPRGER4, CA125, CEA and NSE were observed, reflecting the response differences in patients with stage IV lung cancer patients.

Methylated PTGER4 is capable of predicting the overall survival rate of patients with stage IV NSCLC. To investigate the efficacy of the five markers in predicting long-term prognosis, patients were followed up for $\leq 891$ days and the association between pre- and post-treatment levels, level percentage changes and overall survival rate were calculated. The survival of all patients with lung cancer by predicting the efficacy of all markers, as detailed in Fig. 4. The results indicated that if patients were grouped by the detection threshold of each marker, NSE was the only marker able to predict the overall survival rate at pre-therapy level $(\mathrm{P}=0.026$; $\mathrm{HR}, 0.27$ ), and patients with undetectable pre-therapy NSE levels exhibited a better overall survival rate. Nevertheless, all markers failed to predict the overall survival rate at the post-therapy level. If the median of percentage change was used as the grouping threshold, CA125 $(\mathrm{P}=0.045 ; \mathrm{HR}=0.35)$, CEA ( $\mathrm{P}=0.048 ; \mathrm{HR}, 0.31)$ and NSE $(\mathrm{P}=0.049 ; \mathrm{HR}, 3.01)$ were all able to predict the overall survival rate. Notably, a decrease in marker blood level of CA125 $(<76.0 \%)$ and CEA $(<77.5 \%)$ was associated with a greater survival benefit, while a decrease in NSE level $(<70.0 \%)$ was associated with a shorter survival time. This indicates that a higher NSE expression is associated with a longer survival time.

A total of $76.5 \%(39 / 51)$ of patients in the present study had stage IV non-small cell lung carcinoma (NSCLC), which can be detected using CA125, CEA and Cyfra211 with a high level of sensitivity, hence, the predictive performance of each marker for the overall survival rate time of patients with NSCLC was analyzed. As indicated in Fig. 5, the blood level of pre-therapeutic mPRGER4 ( $\mathrm{P}=0.044 ; \mathrm{HR}, 0.33)$, CA125 $(\mathrm{P}=0.045 ; \mathrm{HR}, 0.28)$ and $\mathrm{NSE}(\mathrm{P}=0.032 ; \mathrm{HR}, 0.14)$ were all capable of predicting the overall survival rate time of patients with NSCLC, and patients with the three markers exhibited improved survival times. Nevertheless, none of the markers were able to predict the post-therapy overall survival rate time. However, if the median of the percentage change was used as the grouping threshold, the level change of CEA $(\mathrm{P}=0.050$; $\mathrm{HR}, 0.29)$ and NSE ( $\mathrm{P}=0.042 ; \mathrm{HR}, 4.14)$ were able to predict overall survival rate times in the present population. Notably, patients with a greater decrease in CEA $(<77.5 \%)$ exhibited longer overall survival rate, while conversely patients with a greater decrease in NSE levels $(<70.0 \%)$ exhibited shorter overall survival rate, supporting the results exhibited in Fig. 4. The changes in the level of Cyfra 211 before and after therapy also showed a potential efficacy of prediction but the results were not significant.

\section{Discussion}

Performance comparison between methylation and protein markers in therapeutic response assessment. Blood protein markers have long been used as a tool for cancer detection. Protein markers were first discovered to have a strong correlation with the occurrence of tumors, but later studies revealed that the majority of protein markers are unable to detect early-stage tumors (17-19). Protein markers are particularly elevated during tumor recurrence and are generally associated 
A

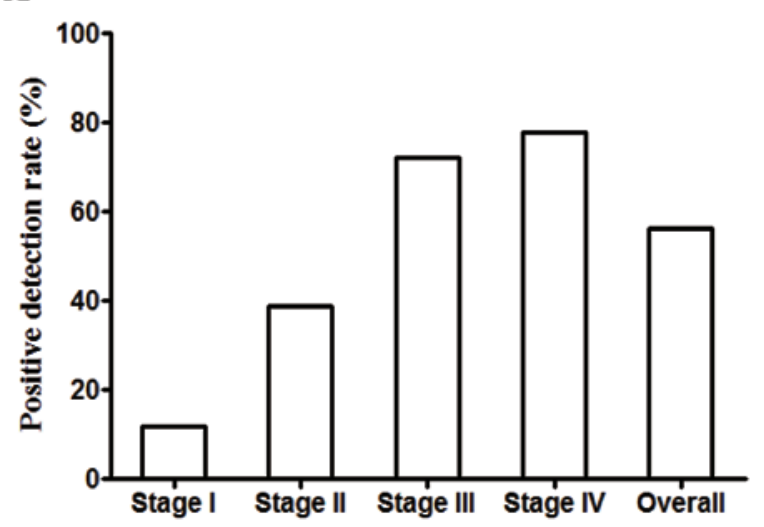

B

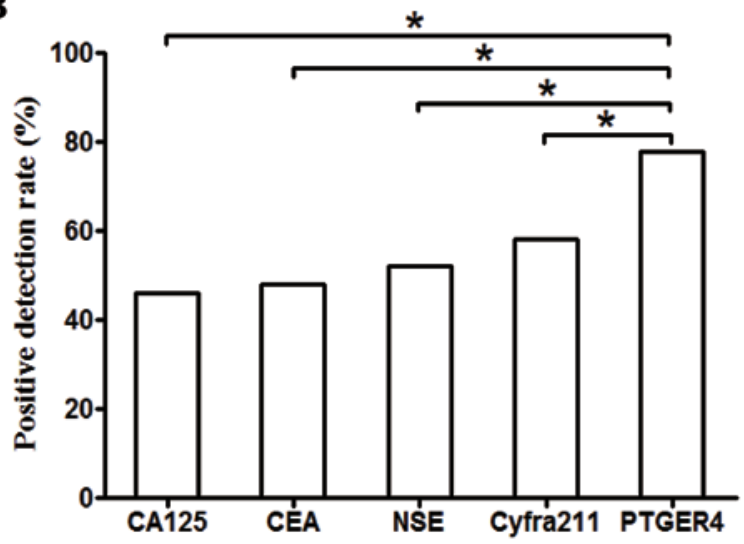

Figure 1. Positive detection rate of methylated PTGER4 in all stages of lung cancer and a comparison with CA125, CEA, Cyfra211 and NSE in stage IV lung cancer. (A) The stage-dependent PDR and overall PDR of methylated PTGER4 in lung cancer detection. (B) Comparison of PDR (sensitivity) between the preand post-therapeutic groups of CA125, CEA, NSE, Cyfra211 with mPRGER4 when the specificity was set to $90 \%$. $\chi^{2}$ test has been performed to compare the PDR and a significant difference ( $\mathrm{P}<0.05)$ was found between PTGERS and the other four markers. PTGER4, prostaglandin E receptor 4; CA125, carcinoma antigen 125; CEA, carcinoembryonic antigen; cyfra21-1, cytokeratin 19-fragment; NSE, neuron-specific enolase; PDR positive detection rate.

MPTGER4
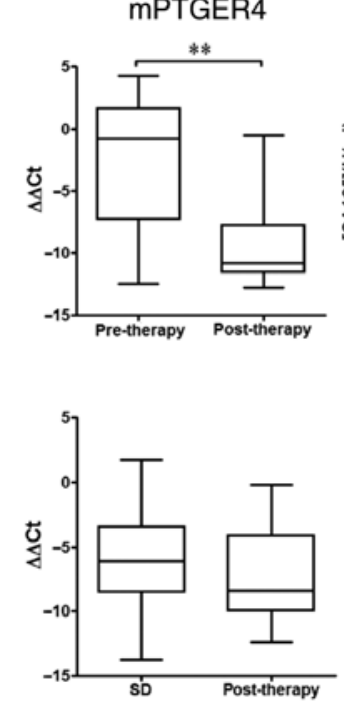

CA125
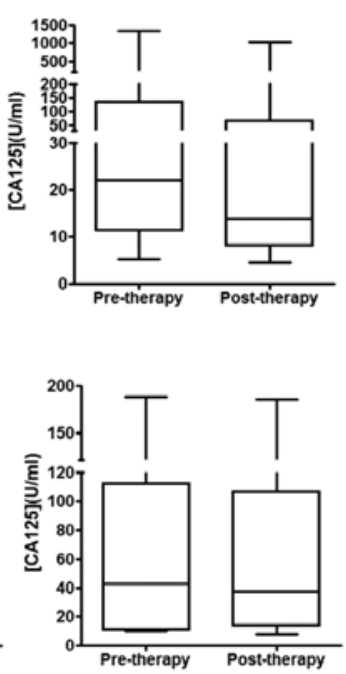

CEA
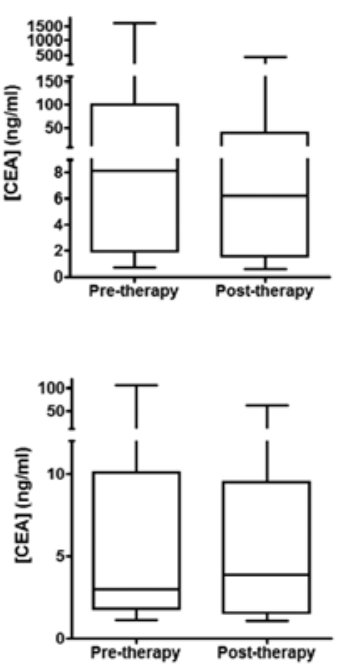

Cyfra211
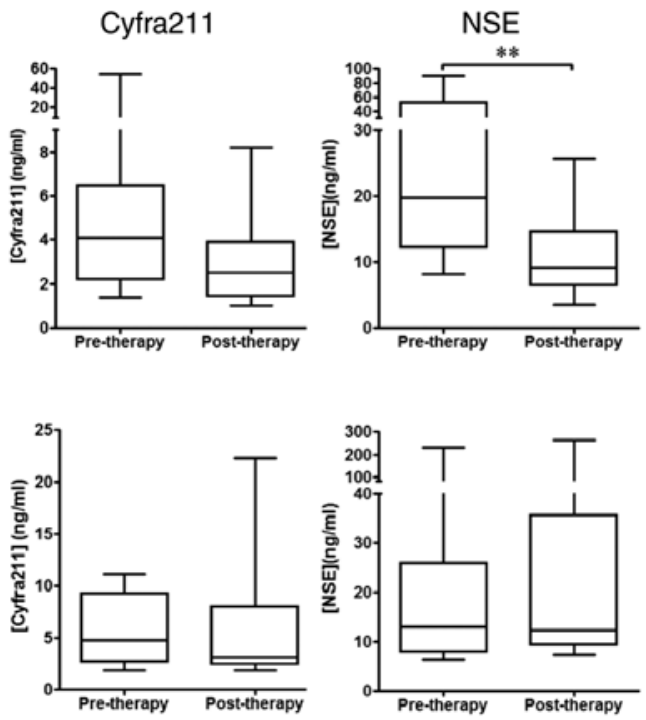

Figure 2. Box plot for the levels of methylated PTGER4, CA125, CEA, Cyfra211 and NSE before and after therapy in patients with stage IV lung cancer. Absolute values of marker blood levels before and after therapy are shown for each marker based on therapeutic responses (PR or SD). The biomarker levels of the pre- and post-therapeutic groups are compared in each panel. ${ }^{* *} \mathrm{P}<0.01$. PTGER4, prostaglandin E receptor 4; CA125, carcinoma antigen 125; CEA, carcinoembryonic antigen; cyfra21-1, cytokeratin 19-fragment; NSE, neuron-specific enolase; PR, partial response; SD, stable disease.

with tumors in more advanced stages (17). Hence, protein tumor markers are predominantly used for recurrence monitoring. An increased level of certain blood protein markers is associated with tumor progression, while a decreased level is an indication of tumor remission (17-19). However, significant changes in blood protein level may not be observed in a group of patients due to variation between individuals. In the present study, no significant difference in CA125, CEA and Cyfra211 levels was observed in the PR group before and after treatment, indicating that protein markers may not be accurate in monitoring therapeutic response. Conversely, methylation markers are more sensitive to tumor burden change compared with protein markers $(15,22,23)$, as they are sensitive to the changes of ctDNA levels in plasma, which reflects the release of cellular DNA during apoptosis or necrosis (24). In the present study, the change in methylated PTGER4 level from pre- to post-treatment was greater than that of the protein markers, indicating that methylated PTGER4 represents a more sensitive marker for therapeutic response assessment. Notably, previous studies have reported the application of methylation markers in monitoring tumor response to therapy $(15,16,22-25)$. The methylation markers chosen for response monitoring share certain common features. Firstly, the pre-treatment sensitivity of the markers is generally satisfactory with a wide coverage of patients. Secondly, whilst having a good sensitivity to tumor burden change, their plasma level changes have a strong association with either disease progression or remission. Thirdly, the level of change is quantifiable and may therefore be able to inform clinical practices $(15,16,22-25)$.

Methylation markers are applicable not only in response monitoring, but also for early diagnosis. Previous studies have suggested that the overall performance of methylation markers 

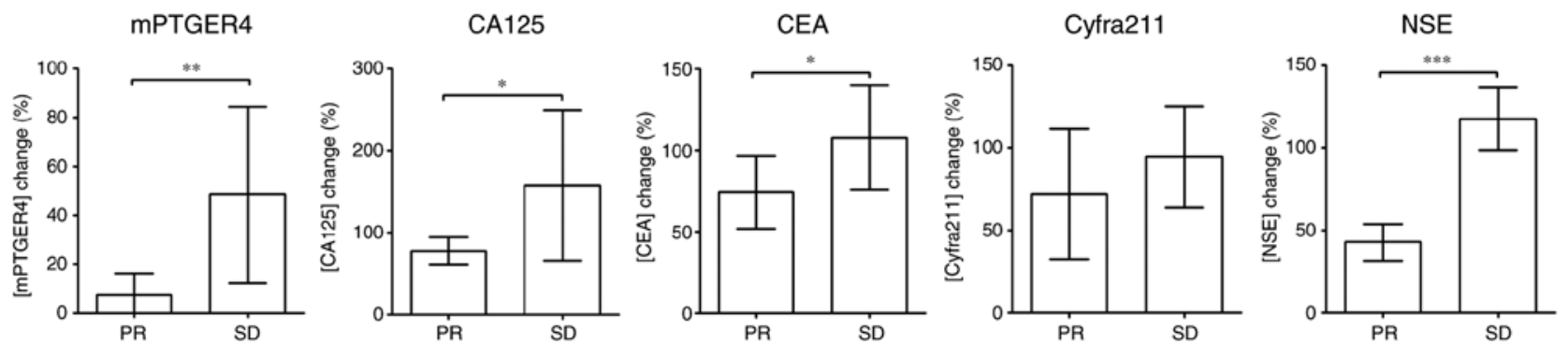

Figure 3. Comparison of level change between PR and SD groups for methylated PTGER4, CA125, CEA, Cyfra211 and NSE. Post-therapeutic marker levels were normalized to pre-therapeutic level for each patient and grouped by therapeutic response (PR or SD). The percentage change of the PR and SD groups are compared in each panel. ${ }^{*} \mathrm{P}<0.05 ;{ }^{* *} \mathrm{P}<0.01 ;{ }^{* * *} \mathrm{P}<0.001$. PTGER4, prostaglandin E receptor 4; CA125, carcinoma antigen 125 ; CEA, carcinoembryonic antigen; cyfra21-1, cytokeratin 19-fragment; NSE, neuron-specific enolase; PR, partial response; SD, stable disease; HR, hazard ratio.
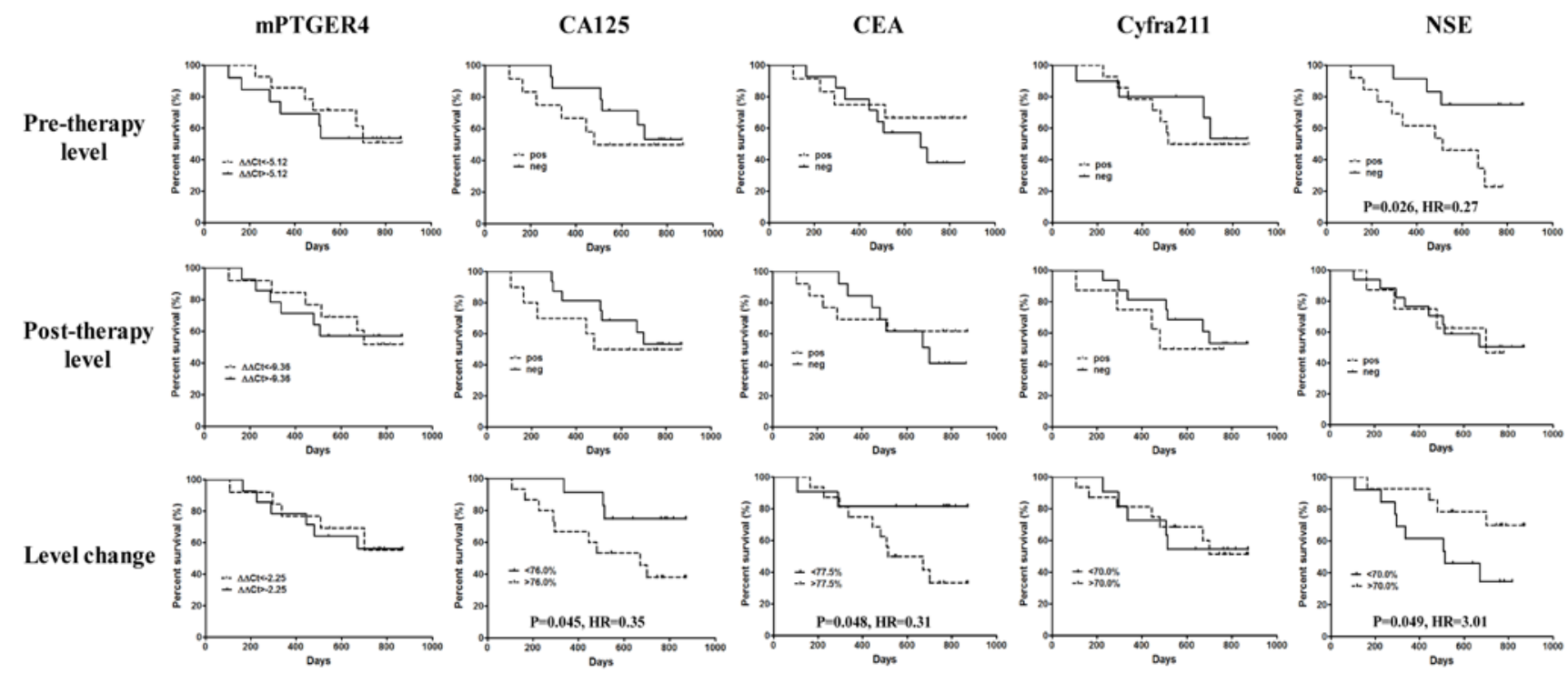

Figure 4. Efficacy of methylated PTGER4, CA125, CEA, Cyfra211 and NSE in predicting the overall survival rate of all patients with stage IV lung cancer in the present study. Pre-therapeutic level, post-therapeutic level and level changes in percentage were used in the prediction for each marker. Comparison has been performed between the two survival curves in each panel. $\mathrm{P}<0.05$ was regarded as a significant difference. PTGER4, prostaglandin E receptor 4; CA125, carcinoma antigen 125; CEA, carcinoembryonic antigen; cyfra21-1, cytokeratin 19-fragment; NSE, neuron-specific enolase; HR, hazard ratio.

in both these applications is an improvement compared with protein markers $(15,16,22-25)$. However, protein markers also have certain advantages. For instance, their specificity for cancer is generally better with a low false-positive rate in healthy subjects, suggesting that the potential of measuring levels of protein markers in the blood requires further investigation (17-19). By contrast, the positive detection rate of methylation markers is generally higher in older patients, with an increased false positive rate in healthy elderly subjects $(26,27)$. Therefore, a combination of methylation and protein markers may be beneficial in enhancing both the sensitivity and specificity of cancer diagnosis and therapeutic monitoring.

Characteristics of markers for predicting long-term survival. Both methylation and protein markers may be useful for predicting long-term survival, but not all of them are effective in predicting overall survival rate. Markers capable of predicting long-term overall survival rate must exhibit the following characteristics: i) The blood level of markers post-treatment must exhibit a significant decrease in patients with PR, and an insignificant change in patients with SD, or a significant increase associated with disease progression; ii) an identifiable association between the marker blood level and the change in tumor size; and iii) the short- or mid-term post-treatment remission of a tumor must have a strong association with the increased long-term survival of patients, while the short- or mid-term tumor progression must also be strongly correlated with the decreased long-term survival of the patients. In the present study, NSE met the above criteria, and its predictive efficacy for survival time was demonstrated in the patients with stage IV NSCLC. The pre-therapeutic level of methylated PTGER4 also exhibited similar efficacy in predicting the overall survival rate of patients with NSCLC. However, the level of methylated PTGER4 decreased in the SD group by $\sim 2.71$ times (the mean $\Delta \Delta \mathrm{Ct}$ decreased from -5.96 to -7.4$)$. This subsequently affected the discrimination of level change between PR and SD patients, interfering with the predictive performance of long-term survival in all stage IV patients. It appeared that SCLC may be a contributing factor that interfered with the predictive ability of mPRGER4. Other previously reported methylation markers, including 

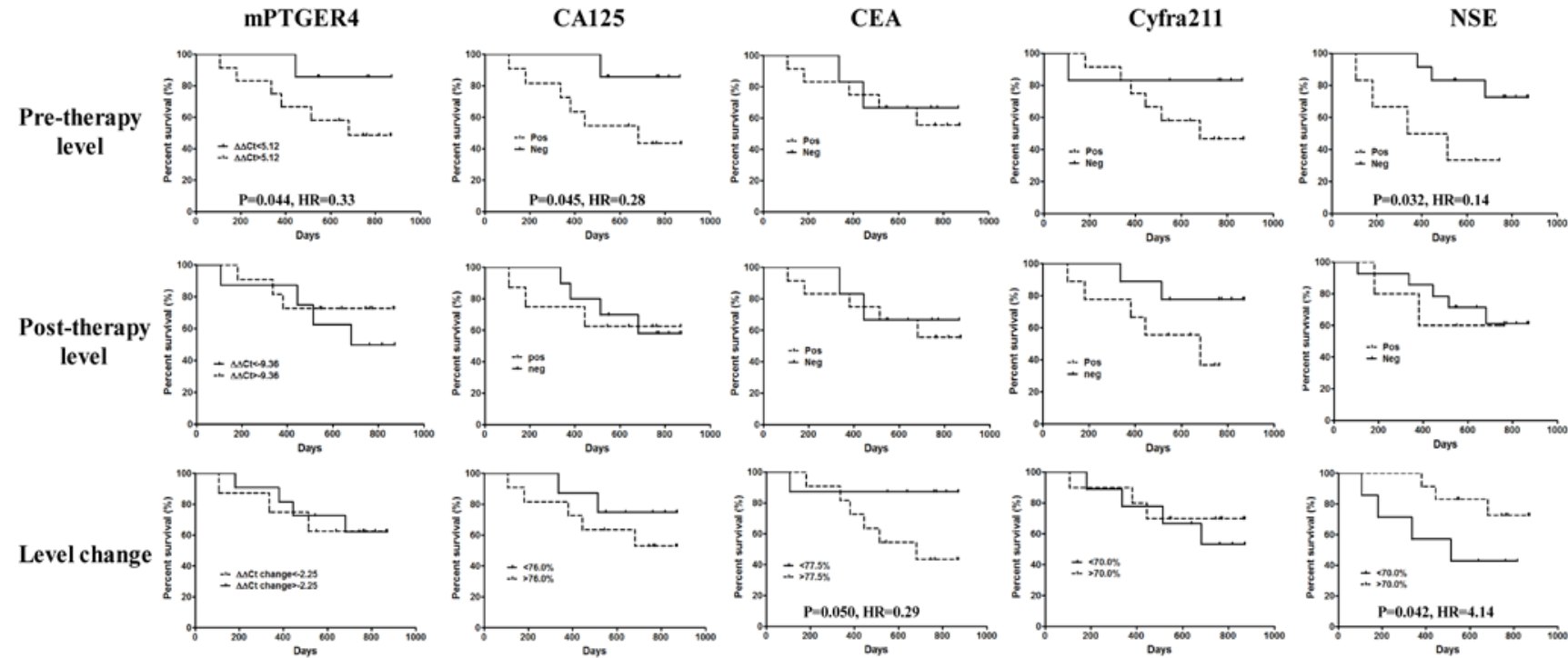

Figure 5. Efficacy of methylated PTGER4, CA125, CEA, Cyfra211 and NSE in predicting the overall survival rate of patients with stage IV non-small cell lung cancer. Pre-therapeutic level, post-therapeutic level and level changes in percentage were used in the prediction for each marker. Comparison has been performed between the two survival curves in each panel. $\mathrm{P}<0.05$ was regarded as a significant difference. PTGER4, prostaglandin E receptor 4; CA125, carcinoma antigen 125; CEA, carcinoembryonic antigen; cyfra21-1, cytokeratin 19-fragment; NSE, neuron-specific enolase.

mSHOX2 $(15,16,20)$ and methylated septin 9 (SEPT9) (22-25), were revealed to be effective in survival prediction in patients with advanced lung or colorectal cancer.

Meanwhile, CA125 during pre-treatment exhibited an ability to predict overall survival rate time in patients with NSCLC. It was also able to predict overall survival rate time of all included patients with stage IV lung cancer. For CEA, its level change had the ability to predict overall survival rate time for all stage IV subtypes and NSCLC specifically. In general, if long-term survival is able to be predicted before treatment, more options and earlier interventions will be available to patients. In the current study, the level of mPRGER4, CA125 and NSE during pre-treatment exhibited clear predictive capability and were therefore more suitable than other markers.

Previous studies have revealed that protein markers that are specific to certain lung cancer types can be used to predict survival (28-34). For instance, lung adenocarcinoma survival can be predicted by measuring CA125 $(29,30)$ and CEA (31) levels, while the survival of patients with squamous cell carcinoma (SC) and SCLC can be predicted by Cyfra211 (32) and NSE levels $(33,34)$, respectively. In the present study, it was revealed that these markers were specific to each cancer type, suggesting the potential application of these markers for the survival prediction of different pathological subtypes accordingly. Furthermore, NSE expression was associated with the survival of patients with NSCLC. Notably, a longer overall survival time was predicted by a higher post-treatment NSE level and the opposite was true of CEA and CA125 levels, which warrants further investigation.

Application of monitoring and predictive biomarkers in clinical settings. Generally, there are two therapeutic options for patients with advanced NSCLC, which depends on the patients' susceptibility to a drug given TKI is normally used as the first-line therapy for patients with TKI-associated drug-sensitive mutations; otherwise, first-line chemotherapy or radiochemotherapy is recommended (13). The second-line treatment is often determined by the patients' condition. Patients who have received first- or second-generation TKI therapy will proceed with third-generation TKI if they have developed resistance induced by specific mutations; otherwise, chemotherapy or immunotherapy are considered (13). Patients with first-line chemotherapy are generally treated with second-line chemotherapy or immunotherapy upon the onset of resistance (13). For patients with SCLC, chemotherapy is currently used as the first-line therapy, with second-line chemotherapy or immunotherapy also considered viable options (35). Furthermore, the FDA has approved the use of immunotherapy as the first-line treatment of NSCLC and SCLC (35). Notably, the treatment of $\mathrm{SC}$ is distinct from that of the ADC due to the differences in tumor biology, symptoms, targeting population and molecular characteristics. SC generally exhibits a much lower rate of mutations in key driver genes, such as EGFR or ALK (36), making chemotherapy as a more suitable first-line therapy compared with TKI-based target therapy in the majority of patients with ADC (13). Second-line chemotherapy and/or immunotherapy are typically suitable for patients with SC who have developed resistance to first-line chemotherapy. Recently, first-line immunotherapy (37) or first-line chemo/immuno combined therapy (38) have been suggested as an improved therapeutic options for patients with late-stage SC.

Further development of therapeutic strategies will provide more options in the treatment of late-stage lung cancer. Regardless of whether the first or multiple lines of therapies were given to patients with ADC, SC or SCLC, current therapies on late-stage lung cancer require intensive real-time monitoring of the patients' condition, as it is necessary to investigate the patients' response before deciding whether to maintain the current treatment or switch to alternative therapies. In the present study, the effects of targeted therapy and 
chemotherapy were evaluated simultaneously, although they are two different types of therapies. This was because the current study focused on the association between therapeutic response and the mPTGER4 level, and the results of the present study support previous reports $(12,13,15)$ which indicate that the level of methylation markers is closely associated with therapeutic response, but not treatment regime. Different treatments exhibit distinct responses that may be reflected by methylation markers; however, treatment type was not the focus of the present study and therefore all therapies were assessed together.

Currently, CT examination is still the most commonly used method, but it is radiative and hence not suitable for repeated use in a short time period. The findings from CT images only reflect a condition that has already happened instead of continuously monitoring the real-time condition, resulting in patients missing out on the best opportunity for treatment. Therefore, response monitoring with blood markers may be an ideal method, but it requires high sensitivity markers that exhibit a good correlation with changes in tumor burden. Previous studies have revealed that both methylation and protein markers can be used for response monitoring, but not all changes in blood marker levels were correlated with tumor burden, and there is a large variation between individuals (17-19). These issues warrant further investigation to characterize the performance of individual markers. In addition, the positive detection rate of methylation markers prior to treatment is typically higher than that of protein markers, making them more suitable for response monitoring.

At present, the performance of a monitoring marker can be assessed according to three aspects: i) Whether it can accurately and timely reflect the real-time changes of a disease; ii) whether it can predict the change or outcome of the disease; and iii) whether it can predict long-term survival and prognosis of a patient. Ideally, one marker or a panel of markers can meet all three aspects simultaneously. The results of disease monitoring are comparable, and a patient's response can be quantified. However, such markers are rare and may not be effective for all patients, therefore, the combination of multiple markers may still be necessary to be sensitive to as many patients as possible.

Future in vivo study and validation on PTGER4 protein expression. It is difficult to build up an in vivo methylation model so far as methylation status varies greatly across different genes and individuals. Since methylation is dynamic and reversible and may be affected by many in vivo factors, its level is hard to evaluate under experimental conditions. Notably, in vivo methylation models targeting certain genes are even more difficult to achieve. Therefore, the majority of methylation studies now use in vitro models, such as cell lines from patients or animals with confirmed methylation status at certain genes. However, the majority of these models represent an ideal situation where the methylation of a certain gene is relatively stable, and the effect of intervention can be assessed and quantified. Examples of studies using these cell line models include a colorectal cancer cell line studying the methylation marker SEPT9 (39), cell lines with abnormal mutL homolog 1 methylation in colorectal cancer and lung cancer (40) and cell lines with abnormal PR/SET domain 2/5/16 gene methylation in lung cancer (41).
There are several limitations in the present study. The number of patients in the present study was small especially when survival analysis was performed on NSCLC patients. Future large-scale studies are needed to verify the conclusions of this study. Furthermore, the methylation change at the individual patient level may not accurately reflect the patient condition, since blood methylation level is affected by many factors. Therefore, decision of therapeutic strategies based on combined information from multiple examinations including methylation, protein markers and imaging methods should be applied to patients. There was a small proportion of patients who cannot be assessed by PTGER4, due to it's sensitivity prior to therapy not being $100 \%$. The therapeutic effect in these patients must be assessed by other methods, such as the use of protein markers or imaging methods (e.g. CT and MRI).

In the present study, the blood methylation level of PTGER4 gene was tested, which was developed as a new marker for discriminating benign from malignant lung nodules (11), while the validation of PTGER4 at protein level has not yet been performed. At present, there is no systematic study comparing the expression level of PTGER4 in lung cancer tissues, para-carcinoma tissues or normal tissues. Theoretically, hypermethylation at the promoter region of PTGER4 in lung cancer may result in decreased expression of PTGER4 protein, but the mechanism is yet to be elucidated. Moreover, the expression level of PTGER4 may also be influenced by changes in other pathways in lung cancer. Therefore, it is also worth exploring the potential affecting factors of PTGER4 expression. Investigation of PTGER4 expression may help to validate the PTGER4 methylation assay and potentially indicate PTGER4 as a new protein marker.

\section{Acknowledgements}

Not applicable.

\section{Funding}

The present study was supported by the Special Funds for Strategic Emerging Industry Development of Shenzhen (grant no. 20170922151538732) and the Science and Technology Project of Shenzhen (grant no. JSGG20180703164202084). Funding bodies did not influence the study design, study implementation, data collection, data analysis, data interpretation and manuscript writing of the present study.

\section{Availability of data and materials}

The datasets used and/or analyzed during the current study are available from the corresponding author on reasonable request.

\section{Authors' contributions}

FZ and LS designed the study. YZ and JH performed the statistical analysis and wrote the manuscript. YZ, JH, QZ, JC, KY, QF, DQ, JW and EB collected the samples, clinical information, diagnostic information and performed the patient follow-up. YZ and $\mathrm{JH}$ analyzed the data. LS proof read the manuscript and submitted the manuscript. All authors read and approved the final manuscript. 


\section{Ethics approval and consent to participate}

The present study was approved by the Ethics Committee of the Affiliated Hospital of Jiangnan University and the eighth medical center of the Chinese People's Liberation Army general hospital. Prospective samples were collected and written informed consent to participate was acquired from the patients.

\section{Patient consent for publication}

Consent for publication was obtained from the participants before the start of the study.

\section{Competing interests}

The authors declare that they have no competing interests.

\section{References}

1. National Lung Screening Trial Research Team, Church TR, Black WC, Aberle DR, Berg CD, Clingan KL, Duan F, Fagerstrom RM, Gareen IF, Gierada DS, et al: Results of initial low-dose computed tomographic screening for lung cancer. N Engl J Med 368: 1980-1991, 2013.

2. Belloni E, Veronesi G, Rotta L, Volorio S, Sardella D, Bernard L, Pece S, Di Fiore PP, Fumagalli C, Barberis M, et al: Whole exome sequencing identifies driver mutations in asymptomatic computed tomography-detected lung cancers with normal karyotype. Cancer Genet 208: 152-155, 2015.

3. Uchida J, Kato K, Kukita Y, Kumagai T, Nishino K, Daga H, Nagatomo I, Inoue T, Kimura M, Oba S, et al: Diagnostic accuracy of noninvasive genotyping of EGFR in lung cancer patients by deep sequencing of plasma cell-free DNA. Clin Chem 61: 1191-1196, 2015.

4. Jin X, Chen Y, Chen H, Fei S, Chen D, Cai X, Liu L, Lin B, Su H, Zhao L, et al: Evaluation of tumor-derived exosomal miRNA as potential diagnostic biomarkers for early-stage non-small cell lung cancer using next-generation sequencing. Clin Cancer Res 23: 5311-5319, 2017

5. Leng Q, Lin Y, Jiang F, Lee CJ, Zhan M, Fang H, Wang Y and Jiang F: A plasma miRNA signature for lung cancer early detection. Oncotarget 8: 111902-111911, 2017.

6. Ye M, Li S, Huang W, Wang C, Liu L, Liu J, Liu J, Pan H, Deng Q, Tang H, et al: Comprehensive targeted super-deep next generation sequencing enhances differential diagnosis of solitary pulmonary nodules. J Thorac Dis 10 (Suppl 7): S820-S829, 2018.

7. Feng Y, Feng G, Lu X, Qian W, Ye J, Manrique CA, Ma C and Lu Y; written on behalf of the AME Lung Cancer Collaborative Group: Exploratory analysis of introducing next-generation sequencing-based method to treatment-naive lung cancer patients. J Thorac Dis 10: 5904-5912, 2018.

8. Oellerich M, Schütz E, Beck J and Walson PD: Circulating cell-free DNA-diagnostic and prognostic applications in personalized cancer therapy. Ther Drug Monit 41: 115-120, 2019.

9. Lim M, Kim CJ, Sunkara V, Kim MH and Cho YK: Liquid biopsy in lung cancer: Clinical applications of circulating biomarkers (CTCs and ctDNA). Micromachines (Basel) 9: E100, 2018.

10. Mayo-de-Las-Casas C, Garzón Ibáñez M, Jordana-Ariza N, García-Peláez B, Balada-Bel A, Villatoro S, Malapelle U, Karachaliou N, Troncone G, Rosell R and Molina-Vila MA: An update on liquid biopsy analysis for diagnostic and monitoring applications in non-small cell lung cancer. Expert Rev Mol Diagn 18: 35-45, 2018.

11. Weiss G, Schlegel A, Kottwitz D, König T and Tetzner R: Validation of the SHOX2/PTGER4 DNA methylation marker panel for plasma-based discrimination between patients with malignant and nonmalignant lung disease. J Thorac Oncol 12: 77-84, 2017.

12. Chu X, Zhao $\mathrm{W}$ and Wang B: DNA methylation detection of SHOX2 and PTGER4 in plasma contributes to differential diagnosis of pulmonary nodule patients. Xi Bao Yu Fen Zi Mian Yi Xue Za Zhi 35: 357-361, 2019 (In Chinese).
13. Ettinger DS, Wood DE, Aisner DL, Akerley W, Bauman J, Chirieac LR, D'Amico TA, DeCamp MM, Dilling TJ, Dobelbower M, et al: Non-small cell lung cancer, version 5.2017, NCCN clinical practice guidelines in oncology. J Natl Compr Canc Netw 15: 504-535, 2017.

14. Livak KJ and Schmittgen TD: Analysis of relative gene expression data using real-time quantitative PCR and the 2(-Delta Delta $\mathrm{C}(\mathrm{T})$ ) method. Methods 25: 402-408, 2001.

15. Schmidt B, Beyer J, Dietrich D, Bork I, Liebenberg V and Fleischhacker M: Quantification of cell-free mSHOX2 Plasma DNA for therapy monitoring in advanced stage non-small cell (NSCLC) and small-cell lung cancer (SCLC) patients. PLoS One 10: e0118195, 2015

16. Peng XM, Liu XL, Xu L, Li Y, Wang H, Song L and Xiao W: The mSHOX2 is capable of assessing the therapeutic effect and predicting the prognosis of stage IV lung cancer. J Thor Dis 11: 2458-2469, 2019.

17. Duffy MJ and O'Byrne K: Tissue and blood biomarkers in lung cancer: A review. Adv Clin Chem 86: 1-21, 2018.

18. Yang B, Li X, Ren T and Yin Y: Autoantibodies as diagnostic biomarkers for lung cancer: A systematic review. Cell Death Discov 5: 126, 2019.

19. Qin J,Zeng N, Yang T, Wan C, Chen L, Shen Y and Wen F. Diagnostic value of autoantibodies in lung cancer: A systematic review and meta-analysis. Cell Physiol Biochem 51: 2631-2646, 2018.

20. Eisenhauer EA, Therasse P, Bogaerts J, Schwartz LH, Sargent D, Ford R, Dancey J, Arbuck S, Gwyther S, Mooney M, et al: New response evaluation criteria in solid tumours: Revised RECIST guideline (version 1.1). Eur J Cancer 45: 228-247, 2009.

21. Epi proLung. Instruction for use. Epigenomics AG, Berlin, 2018. https:// www.epigenomics.com/wp-content/uploads/2018/08/IFU_0018_GB rev4_Instructions_for_Use_Epi_proLung.pdf. Accessed August $\overline{6}$, 2018.

22. Song L, Guo S, Wang J, Peng X, Jia J, Gong Y, Yang B, Xiao W, Dong C, Liu H and Li Y: The blood mSEPT9 is capable of assessing the surgical therapeutic effect and the prognosis of colorectal cancer. Biomark Med 12: 961-973, 2018.

23. Song $\mathrm{L}, \mathrm{Yu} \mathrm{H}$, Jia J and $\mathrm{Li} \mathrm{Y}$ : A systematic review of the performance of the SEPT9 gene methylation assay in colorectal cancer screening, monitoring, diagnosis and prognosis. Cancer Biomark 18: 425-432, 2017

24. Jahr S, Hentze H, Englisch S, Hardt D, Fackelmayer FO, Hesch RD and Knippers R: DNA fragments in the blood plasma of cancer patients: Quantitations and evidence for their origin from apoptotic and necrotic cells. Cancer Res 61: 1659-1665, 2001.

25. Dietrich D, Hasinger O, Liebenberg V, Field JK, Kristiansen G and Soltermann A: DNA methylation of the homeobox genes PITX2 and SHOX2 predicts outcome in non-small-cell lung cancer patients. Diagn Mol Pathol 21: 93-104, 2012.

26. Bergheim J, Semaan A, Gevensleben H, Groening S, Knoblich A, Dietrich J, Weber J, Kalff JC, Bootz F, Kristiansen G and Dietrich D: Potential of quantitative SEPT9 and SHOX2 methylation in plasmatic circulating cell-free DNA as auxiliary staging parameter in colorectal cancer: A prospective observational cohort study. Br J Cancer 118: 1217-1228, 2018.

27. Song L, Jia J, Yu H, Peng X, Xiao W, Gong Y, Zhou G, Han X and $\mathrm{Li} \mathrm{Y}$ : The performance of the mSEPT9 assay is influenced by algorithm, cancer stage and age, but not sex and cancer location. J Cancer Res Clin Oncol 143: 1093-1101, 2017.

28. Song L, Jia J, Peng X, Xiao W and Li Y: The performance of the SEPT9 gene methylation assay and a comparison with other CRC screening tests: A meta-analysis. Sci Rep 7: 3032, 2017.

29. Wang H, Shen L, Geng J, Wu Y, Xiao H, Zhang F and Si H: Prognostic value of cancer antigen -125 for lung adenocarcinoma patients with brain metastasis: A random survival forest prognostic model. Sci Rep 8: 5670, 2018.

30. Isaksson S, Jönsson P, Monsef N, Brunnström H, Bendahl PO, Jönsson M, Staaf J and Planck M: CA 19-9 and CA 125 as potential predictors of disease recurrence in resectable lung adenocarcinoma. PLoS One 12: e0186284, 2017.

31. Grunnet M and Sorensen JB: Carcinoembryonic antigen (CEA) as tumor marker in lung cancer. Lung Cancer 76: 138-143, 2012.

32. Yu Z, Zhang G, Yang M, Zhang S, Zhao B, Shen G and Chai Y: Systematic review of CYFRA 21-1 as a prognostic indicator and its predictive correlation with clinicopathological features in non-small cell lung cancer: A meta-analysis. Oncotarget 8: 4043-4050, 2017

33. Xue F, Zhu L, Wang L and Wang Q. Serum neuron specific enolase levels correlate with patient prognosis for advanced lung cancer. Int J Clin Exp Med 8: 9498-9504, 2015. 
34. Liu X, Zhang W, Yin W, Xiao Y, Zhou C, Hu Y and Geng S: The prognostic value of the serum neuron specific enolase and lactate dehydrogenase in small cell lung cancer patients receiving first-line platinum-based chemotherapy. Medicine (Baltimore) 96: e8258, 2017.

35. Kalemkerian GP, Loo BW, Akerley W, Attia A, Bassetti M, Boumber Y, Decker R, Dobelbower MC, Dowlati A, Downey RJ, et al: NCCN guidelines insights: Small cell lung cancer, version 2.2018. J Natl Compr Canc Netw 16: 1171-1182, 2018.

36. Roviello G: The distinctive nature of adenocarcinoma of the lung. Onco Targets Ther 8: 2399-2406, 2015.

37. Reck M, Rodríguez-Abreu D, Robinson AG, Hui R, Csőszi T, Fülöp A, Gottfried M, Peled N, Tafreshi A, Cuffe S, et al: Updated analysis of KEYNOTE-024: Pembrolizumab versus platinum-based chemotherapy for advanced non-small-cell lung cancer with PD-L1 tumor proportion score of $50 \%$ or greater. J Clin Oncol 37: 537-546, 2019.

38. Paz-Ares L, Luft A, Vicente D, Tafreshi A, Gümüs M, Mazières J, Hermes B, Çay Şenler F, Csőszi T, Fülöp A, et al: Pembrolizumab plus chemotherapy for squamous non-small-cell lung cancer. N Engl J Med 379: 2040-2051, 2018.
39. Ravegnini G, Zolezzi Moraga JM, Maffei F, Musti M,Zenesini C, Simeon V, Sammarini G, Festi D, Hrelia P and Angelini S: Simultaneous analysis of SEPT9 promoter methylation status, micronuclei frequency, and folate-related gene polymorphisms: The potential for a novel blood-based colorectal cancer biomarker. Int J Mol Sci 16: 28486-28497, 2015.

40. Ma Y, Chen Y and Petersen I: Expression and promoter DNA methylation of MLH1 in colorectal cancer and lung cancer. Pathol Res Pract 213: 333-338, 2017.

41. Tan SX, Hu RC, Liu JJ, Tan YL and Liu WE: Methylation of PRDM2, PRDM5 and PRDM16 genes in lung cancer cells. Int J Clin Exp Pathol 7: 2305-2311, 2014.

(i) (9) This work is licensed under a Creative Commons Attribution-NonCommercial-NoDerivatives 4.0 International (CC BY-NC-ND 4.0) License. 